\title{
EDITORIALS
}

\section{Influenza case definitions - optimising sensitivity and specificity}

P Penttinen (Pasi.Penttinen@ecdc.europa.eu) ${ }^{1}$, R Pebody ${ }^{2}$

1. European Centre for Disease Prevention and Control (ECDC), Stockholm, Sweden

2. Public Health England (PHE), London, United Kingdom

Penttinen P, Pebody R. Influenza case definitions - optimising sensitivity and specificity. Euro Surveill. 2015;20(22):pii=21148. Available online: http://www. eurosurveillance.org/ViewArticle.aspx?Articleld $=21148$

Clinical case definitions used for surveillance struggle to satisfy two challenging and potentially conflicting needs - sensitivity and specificity. A more sensitive case definition is useful to estimate disease burden and identify outbreaks. It will identify a larger proportion of the true cases, but often at the cost of finding a large number of cases due to other causes. A more specific case definition, however, will provide a more accurate description of true cases. This is important to assess the evolution of the epidemiology and effect of measures such as vaccination, but often comes at the cost of missing true cases [1].

These needs are reflected in the World Health Organization (WHO) surveillance objectives for influenza surveillance [2].

Epidemiological surveillance of influenza relies on the one hand on a specific case definition of influenzalike-illness (ILI), because many influenza symptoms discriminate poorly from other respiratory or systemic illnesses, and on the other hand on a sensitive case definition, so that the start of the influenza season can be detected accurately and promptly. Experts performing influenza surveillance are engaged in a continuous debate of the appropriate composition of symptoms and signs to be used for ILI surveillance [3]. A non-specific clinical case definition could lead to false alerts for the start of the seasonal influenza epidemic, overestimate the burden of disease or the severity of an epidemic and underestimate the effectiveness of influenza vaccines. Likewise, a non-sensitive case definition could underestimate the severity of the epidemic and might fail to detect outbreaks or unusual epidemiological patterns.

Several influenza case definitions are applied internationally. The European Union (EU) case definition [4], used in the European surveillance of influenza differs from the current WHO [5] and the United States' Centers for Disease Control and Prevention (US CDC) [6] case definitions mainly in setting a specific temperature limit for fever (WHO: $38^{\circ} \mathrm{C}$; CDC $37.8^{\circ} \mathrm{C}$ $\left(100^{\circ} \mathrm{F}\right)$ ). The EU case definition does not require fever or a measured temperature and allows for a larger variety of symptoms, therefore it will be more sensitive and less specific as compared with the WHO and CDC definitions. Due to this, for example, seasonal respiratory syncytial virus (RSV) epidemics are more likely to influence the EU influenza surveillance than the WHO and CDC surveillance.

In this issue of Eurosurveillance Jiang et al. [7] comprehensively assess the performance of surveillance case definitions for ILI recommended by WHO ('old' and 'new' definition), CDC and ECDC. Their exemplary study builds on previous assessments and also includes data on seroconversion of the infected portions of the cohort under study. The authors monitored symptoms and seroconversion in a cohort of 727 adult subjects in Singapore with up to three serum samples taken per subject before and during the 2009 influenza $A\left(\mathrm{H}_{1} \mathrm{~N}_{1}\right)$ pandemic. In total, $13.5 \%$ of the cohort seroconverted and $4.6 \%$ had presumptive influenza episodes. The authors show that the ECDC ILI case definition is more sensitive, but less specific than the ones recommended by WHO or CDC, mainly due to relying on self-reported history of fever rather than a measured temperature. The current, 'new', WHO case definition with fever defined as body temperature $\geq 38^{\circ} \mathrm{C}$ plus cough, has the highest reported positive predictive value (PPV) of the four compared case definitions.

This study is limited in that it has been conducted during a pandemic which affected somewhat different age groups than seasonal influenza usually does. The studied cohort also did not include children, who are heavily affected by both seasonal and pandemic influenza. However, these limitations are unlikely to change the key conclusions suggested by the authors. It is also important to note that the integration of laboratory data based on respiratory sampling of cases can overcome some of the specificity issues and the use of statistical threshold methods, such as the Moving Epidemic Method [8], calculated using comparable, historical 
surveillance data can further optimise the performance of national influenza surveillance systems to meet their objectives.

Nonetheless, the analysis of Jiang et al. provides appropriate additional evidence base to further evaluate the optimal use of current surveillance case definitions for ILI in Europe, the US and globally. National reference laboratories for influenza in the EU/European Economic Association (EEA) Member States are National Influenza Centres within the WHO Global Influenza Surveillance and Response System (GISRS), and are obliged to report influenza cases to both WHO and ECDC. Therefore, the two organisations are engaged in a process to gradually join the surveillance systems to the maximum extent possible. Currently all WHO European region countries report on a regular basis to the The European Surveillance System (TESSy - operated by ECDC) influenza cases from sentinel and other systems and as of the 2014-15 influenza season the European surveillance outcomes are reported by ECDC and WHO online on a joint web-based bulletin [9].

Changing case definitions for surveillance is in most cases a complex process at national and international levels, as they are normally agreed upon in a legislative process. Changing a case definition affects the comparability of data over time, which makes surveillance experts reluctant to change established definitions, unless there is an overriding public health need. The case definitions for ILI surveillance have been discussed extensively among the European experts over the past decade, leading to the adoption of the EU case definition as well as part of adopting the recent WHO case definition. The analysis by Jiang et al. will most likely generate a discussion on the need to review, once again, the case definitions currently in use in Europe at the upcoming annual meeting of the European Influenza Surveillance Network in June.

\section{Conflict of interest}

Both authors are involved in the coordination of the EU influenza surveillance.

Authors' contributions

PP and RP conceived and wrote this editorial with equal contributions.

\section{References}

1. Giesecke J. Modern Infectious Disease Epidemiology. New York: Oxford University Press Inc., 2002.

2. World Health Organization (WHO). Global Epidemiological Surveillance Standards for Influenza. Geneva: WHO. 2014. [Accessed 3 Jun 2015]. Available from: http://www.who.int/ influenza/resources/documents/WHO_Epidemiological_ Influenza_Surveillance_Standards_2014.pdf?ua=1.

3. Aguilera JF, Paget WJ, Mosnier A, Heijnen ML, Uphoff H, van der Velden J, et al. Heterogeneous case definitions used for the surveillance of influenza in Europe. Eur J Epidemiol. 2003;18(8):751-4. http://dx.doi.org/10.1023/A:1025337616327 PMID:12974549
4. European Centre for Disease Prevention and Control (ECDC). Influenza case definitions. Stockholm: ECDC. [Accessed 3 Jun 2015.] Available from: http://ecdc.europa.eu/en/activities/ surveillance/eisn/surveillance/pages/influenza_case definitions.aspx.

5. World Health Organization (WHO). WHO surveillance case definitions for ILI and SARI. Geneva: WHO. January 2014. [Accessed 3 Jun 2015.] Available from: http:// www.who.int/influenza/surveillance monitoring/ ili_sari_surveillance_case_definition/en/.

6. Centers for Disease Control and Prevention (CDC). Overview of Influenza Surveillance in the United States. Atlanta: CDC. 27 Jan 2015. [Accessed 3 Jun 2015.] Available from: http://www. cdc.gov/flu/weekly/overview.htm.

7. Jiang L, Lee VJ, Lim WY, Chen MI, Chen Y, Tan L, et al. Performance of case definitions for influenza surveillance. Euro Surveill. 2015;20(22):21145. Available from: http://www. eurosurveillance.org/ViewArticle.aspx?Articleld=21145

8. Vega T, Lozano JE, Meerhoff T, Snacken R, Mott J, Ortiz de Lejarazu R, et al. Influenza surveillance in Europe: establishing epidemic thresholds by the moving epidemic method.

Influenza Other Respi Viruses. 2013;7(4):546-58. http://dx.doi. org/10.1111/j.1750-2659.2012.00422.x PMID:22897919

9. European Centre for Disease Prevention and Control (ECDC) / World Health Organization (WHO) Regional Office for Europe. Flu News Europe. Stockholm / Copenhagen: ECDC / WHO Europe. [Accessed 3 Jun 2015]. Available from: https://www. flunewseurope.org/ 